book, there is no doubt that experience and knowledge in and around the UN System were well represented. However, if the author calls the group international, this cannot mean the adequate representation of differing national and cultural views. Rather, the book has to be regarded as a Western approach to explain the situation of the world organization. As such, it is a valuable guide through the current system of the so-called "UN family".

Ulf Marzik

\title{
Dieter Blumenwitz
}

Einfuihrung in das anglo-amerikanische Recht

3. Aufl. 1987, 133 S., Verlag C. H. Beck, München, DM 24,-

Man mag es drehen und wenden: Ohne Grundkenntnisse im anglo-amerikanischen Recht kommt heute weder der forschende noch der praktizierende Internationalrechtler aus. Für die Qualität des Einstiegs, den Blumenwitz vermittelt, spricht die nunmehr dritte Auflage seiner kurz und kompakt gefaßten Einführung. Sie will weniger die materiellrechtlichen als vielmehr die methodisch-handwerklichen Grundlagen des anglo-amerikanischen Rechtskreises vermitteln. Dies gelingt ihr ohne Zweif el, zumal sie mit weiterführenden und vertiefenden Hinweisen opulent bestückt ist. Gleichwohl ist auch etliches Wesentliche über Rechtshistorie und gegenwärtige Entwicklung des materiellen Rechts zu erfahren. Eine gewinnbringende Anschaffung.

Karl-Andreas Hernekamp

\section{Kurt Rabl/Christoph Stoll/Manfred Vasold}

From the U.S. Constitution to the Basic Law of the Federal Republic of Germany. Von der amerikanischen Verfassung zum Grundgesetz der Bundesrepublik Deutschland Verlag Moos \& Partner, Gräfeling 1988; 200 S., DM 36,-

Geht man von dem Titel und dem kurzen Vorwort aus, könnte der Leser von diesem Buch eine Darstellung der geistesgeschichtlichen Entwicklung erwarten, die vor nun 200 Jahren zur amerikanischen Verfassung und vor bald 40 Jahren zum Grundgesetz der Bundesrepublik Deutschland geführt hat; eine Darstellung, bei der die gegenseitige, kontinent-übergreifende Einflußnahme im Vordergrund stehen würde. Dieser Erwartungshaltung wird es jedoch nicht gerecht. Bei näherer Betrachtung stellt man fest, daß es ihr auch gar nicht gerecht werden kann: Von den 200 Seiten werden allein 40 Seiten von 
dem umfangreichen Anhang (Text der Unabhängigkeitserklärung, der amerikanischen Verfassung, auszugsweise der Weimarer Verfassung und des Grundgesetzes) in Anspruch genommen. Der verbleibende Text ist reich bebildert (insgesamt 241 Dokumente und Abbildungen) und zudem in Form der Parallel-Ausgabe zweisprachig verfaßt. Dadurch reduziert sich der für die textliche Darstellung zur Verfügung stehende Raum erheblich. Wer also eine wissenschaftliche Auseinandersetzung mit der Thematik erwartet, wird hierin vergeblich danach suchen. Auch als "Buch zum Nachschlagen" (so der Text der Rückseite) ist es höchstens in seinem Anhang geeignet. Dieses Buch ist ein (Bilder-)Lesebuch, das einen ersten kurzen Uberblick über die jeweilige Entwicklung auf dem neuen und dem alten Kontinent vermitteln kann.

Es ist in drei Abschnitte gegliedert, die jeweils von einem der drei Autoren verfaßt wurden. Zu Beginn befaßt sich Kurt Rabl mit der Entwicklung der ersten Gemeinwesen der Auswanderer, über ihre Lossagung von England bis zum Inkrafttreten der amerikanischen Verfassung. In aller Kürze verdeutlicht er darin die inneren Bedingungen, unter denen sich diese Entwicklung vollzog: das ursprüngliche Bemühen um die Wahrung englischer Tradition und den Wandel zur Begründung einer eigenen, einer amerikanischen. Dabei kommen kritische Töne leider etwas zu kurz: Die Tatsache z.B., daß auch auf dem amerikanischen Kontinent Diskriminierungen (nach Rasse, in einigen Staaten nach Kirchenzugehörigkeit oder aufgrund von Eigentumsklauseln im Wahlrecht) an der Tagesordnung waren, hätte ruhig etwas stärker betont werden können, ohne daß dadurch die Leistung, die in der Erarbeitung der amerikanischen Verfassung und der Bill of Rights liegt, geschmälert worden wäre. Auch wird der Autor der selbstgestellten Aufgabe, "Wechselwirkungen zwischen amerikanischem und deutschem Verfassungsdenken und -recht während der vergangenen beiden Jahrhunderte nachzuzeichnen" (S. 17) nicht gerecht. Im wesentlichen beschränkt sich das Kapitel "Deutsche Beiträge zu amerikanischen Entwicklungen im 17. und 18. Jahrhundert" auf die knappe Darstellung von Einzelschicksalen dreier Deutscher (Jacob Leisler, Franz Daniel Pastorius, Johann Peter Zenger) im Amerika der damaligen Zeit.

Im Anschluß an einen eingeschobenen Exkurs über die wichtigsten Daten der französischen Revolution (der leider mit keinem Wort zu den übrigen Beiträgen in direkte Beziehung gesetzt wird) geht Christoph Stoll auf die Entwicklung in Deutschland ein. Ausgehend vom Wiener Kongreß und der damaligen Gründung des Deutschen Bundes gibt er zunächst einen kurzen Uberblick über die Verfassungsentwicklung innerhalb des Bundes bis 1848. Angesichts der Fülle der Ereignisse in dieser Zeit und der komprimierten Darstellungsweise bleibt dieser notwendigerweise sehr kursorisch. Dennoch kommt die Spannung, die in den unterschiedlichen Verfassungslagen in den nord- und den süddeutschen Ländern, den Vorgängen zwischen Wartburgf est, Juli-Revolution und Hambacher Fest, den ständigen Wechselwirkungen zwischen Reaktion und Opposition lag, anschaulich zum Ausdruck.

Sein Hauptaugenmerk richtet Stoll auf die sich an die Revolution von 1848 anschließende Verfassungsarbeit in der Paulskirche. Deutlich werden dabei insbesondere die Ein- 
flüsse, die von der amerikanischen Verfassung auf die Nationalversammlung einwirkten und die trotz der völlig unterschiedlichen politischen Lage beider Länder in den maßgeblichen Bestimmungen der Reichsverfassung wiederzufinden sind. Die Gründe für das Scheitern dieser Verfassung, ebenso wie die weiteren Ereignisse bis zur Gründung des Deutschen Reichs 1871, werden dagegen leider wieder nur kurz angesprochen.

Im dritten Teil stellt Manfred Vasold die amerikanische und die deutsche Entwicklung auf sozialem, wirtschaftlichem und rechtlichem Gebiet in der Zeit nach 1860 einander ausschnittweise gegenüber. Dabei setzt er zu Beginn die Ereignisse des amerikanischen 'Civil War' mit der deutschen Entwicklung vom Deutschen Bund zum Deutschen Reich unter dem Titel "Erneuerung des Bundes" in rechtlicher Hinsicht gleich. Dies erscheint äußerst zweifelhaft, da er damit den Ubergang vom Staatenbund zum Bundesstaat auf deutscher Seite, d.h. von der Völkerrechtsordnung zur innerstaatlichen Rechtsordnung, ignoriert.

Zu Recht weist der Autor aber auf eine Gemeinsamkeit in anderer Hinsicht hin: Beide Länder befanden sich zu dieser Zeit bezogen auf ihre industrielle Entwicklung auf etwa gleichem (zurückgebliebenen) Niveau, was er anhand einer Reihe von Daten belegt. Das sich anschließende schnellere Wachstum in den USA zeigte dort eine gewaltige gesellschaftliche Sprengwirkung und führte zu einer Vertiefung der sozialen Ungleichheit. Der Autor legt dar, wie der Supreme Court aufgrund der nur lückenhaften Regelungen in der Verfassung in dieser Zeit tatkräftig in das politische Geschehen eingriff: im Regelfall zugunsten der Starken und der Freiheit, die es sich versagt, den Mächtigen Zügel anzulegen. "Die Amerikaner wendeten Darwins Theorie vom Úberleben bevorzugt auf die Gesellschaft an" (S. 108). Hier kommen in detaillierter Betrachtung Aspekte der Verfassungswirklichkeit zur Sprache, die im übrigen in diesem Buch bedauerlicherweise nur in Ansätzen zu finden sind.

Für-das Deutschland der 1890er Jahre begnügt sich der Autor mit dem Hinweis, daß die Arbeiter trotz der ersten Schritte in Richtung Sozialstaat (Sozialversicherungsgesetze) ein politisch unbehaupteter Stand blieben - nach wie vor herrschte dort eine "agrarischfeudale, vorindustrielle Elite" (S. 111).

In bezug a uf die Entwicklung im 20. Jahrhundert stellt Vasold u.a. die Bemühungen der USA dar, in deutsche Verfassungsfragen gestaltend einzugreifen: zuerst mit dem Ende des Ersten Weltkriegs, als Wilson die Demokratisierung und Parlamentarisierung Deutschlands zu einer Vorbedingung des Waffenstillstands machte (daß der Autor seine Feststellung, Deutschland sei ohnehin bereit gewesen, fortan als parlamentarische Demokratie zu bestehen [S. 115], nicht ganz so kategorisch meint, wie sie klingt, zeigt sich daran, daß er später dessen veraltete Gesellschaftsstruktur und die politische Unreife der Deutschen mit als Ursachen für das Scheitern 1933 nennt). Umfassender geht er dann auf diese Bemühungen nach dem Zweiten Weltkrieg ein. Dabei betont er insbesondere, daß die USA im Vergleich zu den übrigen Westmächten am stärksten dazu bereit waren, in ihrer Zone ein demokratisches Eigenleben der Deutschen zu fördern. 
Das letzte Kapitel, das sich unter dem Titel "Bonn und Washington" mit der Zeit nach dem Erlaß des Grundgesetzes befaßt, leidet sehr unter seiner extremen Kürze. Die einzelnen Gesichtspunkte, die der Autor herausgreift (z.B. das Spannungsverhältnis zwischen Verfassungsanspruch und -wirklichkeit, wie es in der Bundesrepublik in der Diskussion über die Notstandsgesetzgebung und den sog. Radikalenerlaß deutlich wurde und in den USA in dem Machtzuwachs der Exekutive auf Kosten der Legislative - als Beispiele nennt er hier Watergate und die Iran-Contra-Affäre) sind jeweils für sich genommen ganz wesentlich für das Verständnis der Gegenwart. Sie bleiben hier jedoch lediglich aneinandergereihte Stichpunkte und können aufgrund der notwendigerweise subjektiven Auswahl auch in ihrer Gesamtschau kein umfassendes Bild vermitteln.

Insgesamt positiv zu bewerten an diesem Buch ist das bereits erwähnte reichhaltige Bildund Dokumentationsmaterial, das auch so manche inhaltliche Ergänzung gibt. Besonders hilfreich ist die zweisprachige Parallelfassung der Originaldokumente im Anhang, originell der Faksimileabdruck der Unabhängigkeitserklärung aus einer deutsch- und einer englischsprachigen Zeitschrift aus Pennsylvania von 1776. Dagegen ist es ärgerlich, daß die englische und die deutsche Textfassung im übrigen an einigen Stellen inhaltlich voneinander abweichen. Auch sind Fachtermini z.T. direkt übersetzt, anstatt daß sie sinngemäß in der anderen Sprache umschrieben wären, was Raum für Mißverständnisse läßt. Warum einzig die Fußnoten ausschließlich auf deutsch abgefaßt sind, ist unerklärlich.

Yorck Jetter 